\title{
DUST IN EARLY TYPE GALAXIES OBSERVED AT THE CFHT
}

\author{
J. Kormendy ${ }^{1}$ and J. Stauffer \\ Dominion Astrophysical Observatory
}

A program of CCD imaging of early-type galactic nuclei carried out at the Canada-France-Hawaii Telescope (Kormendy, these proceedings) reveals dust in an unusually large fraction of the galaxies. Often the dust is in a ring or disk whose radius is comparable to the core radius $r_{c}$; since $r_{c}$ is small, our detection rate benefits from the excellent CFHT seeing (median FWHM $=0$ !'7; scale $=0$ !'22 pixel $^{-1}$ for an RCA CCD used at the Cassegrain focus). We plan to prepare a photographic atlas of unsharp-masked images, and to study correlations between dust properties and nuclear emission lines, $\mathrm{HI}$ and X-ray gas content, radio continuum jets and the overall light distributions of the program galaxies.

Two techniques of "unsharp masking" are used to illustrate dust distributions. In each case the galaxy image is divided by a dust-free model image. The simplest model image is a smoothed version of the original: each pixel is replaced with the mean in a square box surrounding it. Alternatively, we calculate the galaxy profile using the VISTA image processing system and construct a model galaxy having this profile and exactly elliptical isophotes. The first technique (Fig. 1d) is preferable for dust rings, the second (Fig. 1a-c) for radial or large-scale dust features.

Figure 1 shows some of the variety of dust structures seen. The panels are arranged in order of increasing regularity, from chaotic dust structure in an HI elliptical that may be a recent merger remnant (Raimond et al. 1978) to more "settled" distributions in NGC 1199 and the two radio-jet galaxies. The good CFHT seeing has allowed us to detect significant amounts of dust in many galaxies where it was not previously known (e. g. NGC 4621, NGC 4636, NGC 5846). For example, nearly half of King's (1978) sample of normal ellipticals show dust.

Dust lanes are especially common in radio galaxies. The relative alignment of the dust lane and the optical major axis is a diagnostic of the 3-D shape of the galaxy; comparison of the dust lane orientations with the radio jet axes has implications for the formation of the jet and its interaction with the local ISM. Figure 2a shows the comparison between dust and optical major-axis position angle; the tendency for $\triangle \mathrm{PA}$ (dust-opt) to have values near $0^{\circ}$ and $90^{\circ}$ suggests that most ellipticals are oblate while a few may be prolate. Like Kotanyi and Ekers (1979), we find that $\triangle \mathrm{PA}$ (dust-jet) tends to have values near $90^{\circ}$. This may be expected for jets produced by accretion disks, although jet/disk models do not require the ISM at large radii (where we observe it) to be aligned with the dust at small radii.

${ }^{1}$ Visiting Astronomer, CFHT, operated by NRC(Canada), CNRS(France), and the University of Hawaii.

\section{REFERENCES}

King, I. 1978, Ap. J., 222, 1.

Kotanyi, C. and Ekers, R. 1979, A. A., 73, L1.

Raimond, E., Faber, S., Gallagher, J., and Knapp, G. 1978, Ap. J., 246, 708. 


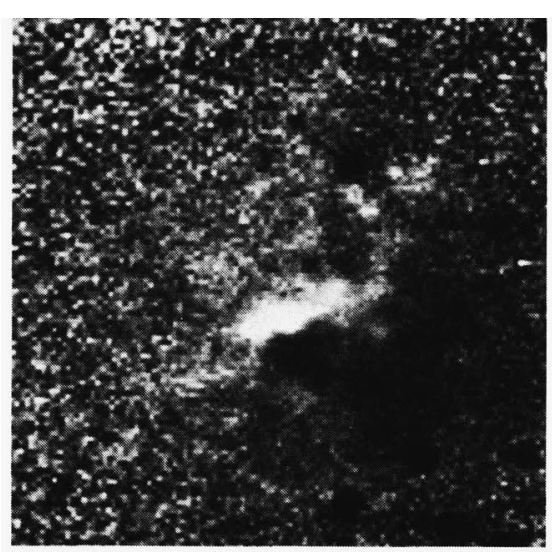

NGC 4636

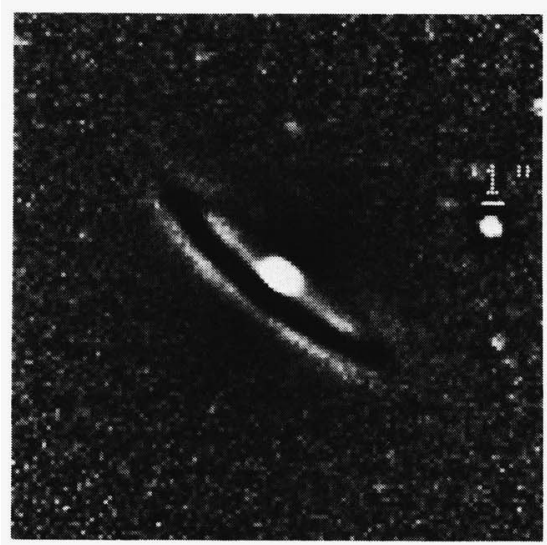

NGC 1199

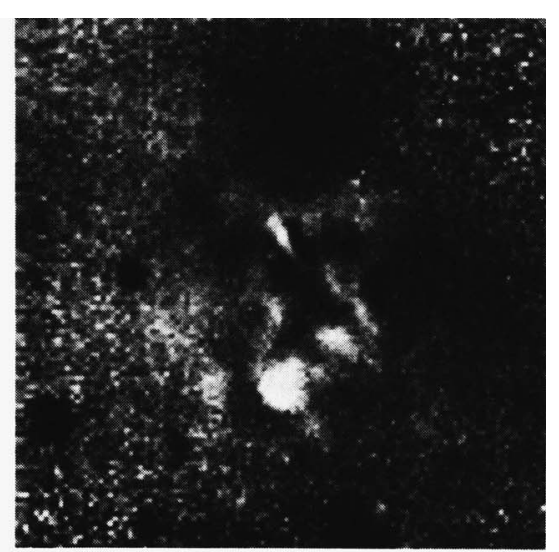

NGC 315

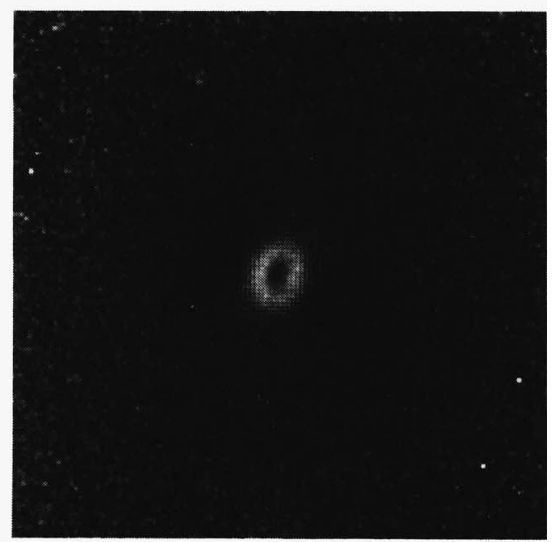

NGC 4261

Figure 1 (above): Unsharp-masked images of four galaxies observed at the CFHT. Figure 2 (below): Histograms of relative position angles of dust lanes, radio jets and optical major axes.
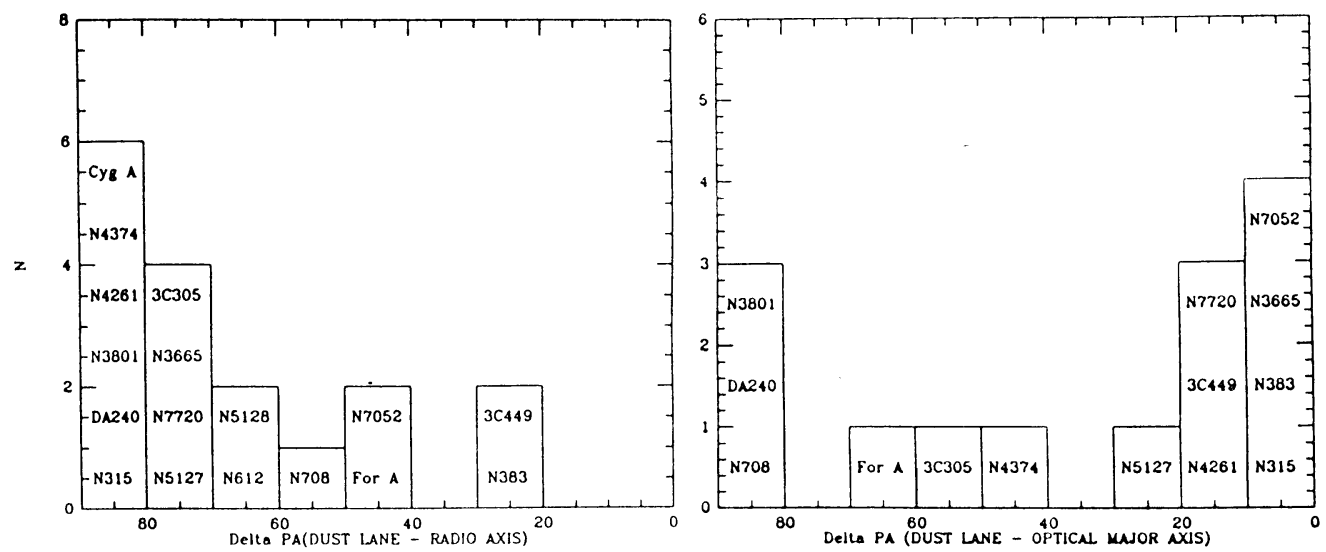